\title{
Análise da vulnerabilidade das edificações em áreas afetadas por movimentos de massa
}

\section{Assessment of building vulnerability in areas affected by mass movements}

\author{
Tamiris Lopez Ferreira*1 $₫\left(\mathbb{D}\right.$, Tiago Damas Martins ${ }^{2} \bowtie$ (D) \\ ${ }^{1}$ Instituto de Ciências Ambientais, Químicas e Farmacêuticas, Universidade Federal de São \\ Paulo, São Paulo, SP, Brasil \\ 2Instituto das Cidades, Universidade Federal de São Paulo, São Paulo, SP, Brasil \\ E-mail: td.martins@unifesp.br \\ *E-mail para correspondência: lopez.tamiris@unifesp.br
}

Resumo: A ocorrência dos movimentos de massa pode assumir distintas grandezas diante do número de mortes e dos severos danos sociais e ambientais para a região afetada. Diferentes esforços têm sido empregados para avaliar os impactos destes processos e dentre eles estão os métodos de cartografia de risco como o mapeamento da vulnerabilidade das edificações. O objetivo do trabalho é analisar a vulnerabilidade das edificações e sua distribuição espacial na área urbana do município de Itaoca (SP) afetado por fluxo de detritos e lama em janeiro de 2014. Foi empregado o método PTVA (Papathoma Tsunami Vulnerability Assessment) sendo adotados os seguintes critérios: número de pavimentos, distância do canal $(<30 \mathrm{~m}$ ou $>30 \mathrm{~m}$ ), existência de muro de alvenaria e tipo de material. Os resultados revelaram que a maioria das edificações tem um valor alto de vulnerabilidade $(>0,83)$, sendo que a ausência de muros de alvenaria é o critério de maior peso. A espacialização registrou uma Baixa Vulnerabilidade para um pouco mais de $14 \%$ da área, e Muito Alta Vulnerabilidade para aproximadamente 10\%. As classes Média e Alta marcaram 39\% e $35 \%$.

Palavras-chave: Desastres Naturais; Fluxos de Detritos; PVTA; Itaoca (SP).

Abstract: The occurrence of mass movements may assume different magnitudes given the number of deaths and the severe social and environmental damage to the affected region. Many efforts have been employed to assess the impacts of these processes and among them are risk mapping methods such as mapping buildings vulnerability. Thus, this work aimed to assess the vulnerability of buildings and their spatial distribution in the urban perimeter of the municipality of Itaoca (SP) affected by mud and debris flows in January 2014. For that, the PTVA method (Papathoma Tsunami Vulnerability Assessment) was applied based on the following criteria: the number of floors, distance from the channel $(<30 \mathrm{~m}$ or $>30 \mathrm{~m})$, the existence of masonry wall, and type of construction material. The results revealed that most buildings have a high vulnerability value (>0.83), with the absence of masonry walls having this criterion the main role on the results. Spatialization registered a Low Vulnerability for nearly $14 \%$ of the area and a Very High Vulnerability for approximately 10\%. The middle and upper classes scored 39\% and 35\%.

Keywords: Natural disasters; Debris flows; PVTA; Itaoca (SP).

\section{Introdução}

Os movimentos de massa podem assumir distintas intensidades e levar a eventos de desastre, notadamente diante do número de mortes e dos severos danos sociais e ambientais para a região afetada por esse tipo de processo geomorfológico. Dados organizados na plataforma Emergency Events Database (EM-DAT) e expressos no relatório Economic Losses, Poverty and Disasters de autoria do Centre for Research on the Epidemiology of Disasters - CRED (2018) revelam que no período entre 1998 até 2017, 5,2 \% do total de eventos de desastre, no mundo, estiveram relacionadas a esse tipo de processo, afetando 4,8 milhões de pessoas. Particularmente no Brasil, um estudo do IBGE (2018) feito a partir da Base Territorial Estatística de 
Áreas de Risco (BATER) estima que 8,3 milhões de pessoas vivam em áreas de risco em 872 municípios. Deve-se destacar ainda o levantamento feito por Macedo e Martins (2015) que apontou que entre 1988 até 2015, 243 municípios brasileiros foram afetados por esses processos, resultando na morte de 3.396 pessoas.

Dentre os diversos eventos ocorridos no Brasil, o relevo montanhoso da Serra do Mar é uma paisagem com frequentes registros, com destaque para a ocorrência na Serra das Araras (RJ) e em Caraguatatuba (SP) em 1966/1967, em Santa Catarina em 2008, no município de Angra dos Reis (RJ) em 2010, nos estados do Rio de Janeiro e Paraná em 2011 e em Itaoca (SP) em 2014.

No verão de 1966/1967, a cidade de Caraguatatuba registrou um acumulado de precipitação pluvial de $586 \mathrm{~mm} / 48 \mathrm{~h}$, o que deflagrou centenas de escorregamentos nas encostas e fluxos de detritos em diversas bacias hidrográficas da fachada Atlântica da Serra. O material mobilizado atingiu a rede de drenagem e alcançou a área urbana do município, causando a morte de 120 pessoas e a destruição de 400 casas, além de danos à infraestrutura urbana (De PLOEY; CRUZ, 1979; DIAS et al., 2016).

Casos mais recentes, como o ocorrido em alguns municípios de Santa Catarina em novembro de 2008, deixou mais de 80.000 desalojados e 85 municípios em estado de emergência, acarretando a queda na produção da indústria, na interrupção do abastecimento de gás e perdas no turismo (VIEIRA; GRAMANI, 2015). Em março de 2011 houve registro de escorregamentos e fluxos de detritos e inundações bruscas nos municípios de Antonina, Morretes e Paranaguá, estado do Paraná, sendo a Comunidade de Floresta uma das áreas mais afetadas, com a destruição total de 11 casas, além de danos na infraestrutura viária (PICANÇO; NUNES, 2013; ZAI et al., 2017).

No Vale do Rio Paraitinga, em 2010, movimentos de massa e inundações levaram a destruição de parte do patrimônio histórico do município de São Luiz do Paraitinga (SP) (BROLLO et al., 2010; BONINI et al., 2021) e no Vale do Ribeira em 2014, movimentos de massa e inundações bruscas atingiram os municípios de Itaoca e Apiaí (SP) danificando ao menos 565 edificações (MATOS et al., 2018).

Diante desses episódios, vários métodos têm sido propostos e empregados buscando uma melhor compreensão da dinâmica dos processos, os fatores condicionantes para a sua ocorrência e os fatores de deflagração (FERNANDES et al., 2001). Nesse sentido, um conjunto de conceitos dão suporte para essas análises, como evento que trata de definir o momento e a área de alcance de um fenômeno que pode, ou não, ser danoso; o perigo, associado a uma condição potencial de um fenômeno promover algum tipo de resultado prejudicial; a suscetibilidade que é uma condição potencial do terreno para a ocorrência do processo; a vulnerabilidade que está atrelada a um nível de perda quando da ocorrência de um evento, e o risco que revela uma relação entre a potencial ocorrência de um evento e o dimensionamento da magnitude do evento desastroso (BRASIL, 2007); sendo que o entendimento trazido nesses conceitos levam a definição de uma área de risco (CERRI et al., 2007).

Particularmente, os métodos que buscam avaliar a vulnerabilidade de um elemento exposto, visam quantificar um nível de vulnerabilidade para esse elemento em uma condição de perigo, no caso de ocorrência de um evento potencialmente danoso, havendo variação no objeto avaliado (ex.: grupo de pessoas ou um conjunto material) e nos critérios, como características socioeconômicas de uma população ou padrões construtivos de edificações.

Matos et al. (2017) avaliaram a vulnerabilidade de edificações tomando como base as características dos padrões construtivos, tipo de material utilizado (ex.: alvenaria ou madeira) e a localização em relação ao alcance de fluxo de detritos para o município de Caraguatatuba (SP), a partir da avaliação espacial dos depósitos resultantes desses fluxos no evento de 1966/1967, mencionado anteriormente. Já GuillardGonçalves e Zêzere (2018) realizaram uma análise combinada entre vulnerabilidade social dos residentes e a vulnerabilidade física das edificações para o município de Loures (Portugal), concluindo que 0,9\% da população reside em $75 \%$ de área suscetíveis a ocorrência de movimentos de massa (escorregamentos). Neste caso, os autores assumem que a vulnerabilidade social é uma razão entre a sensibilidade populacional e a falta de resiliência da comunidade. Um outro exemplo é o trabalho de Dias et al. (2020) que propuseram um índice para análise da vulnerabilidade social incorporando dados socioeconômicos de 443 municípios brasileiros. Os autores concluíram que $42 \%$ da população avaliada está em uma condição de alta vulnerabilidade.

Os esforços demonstrados nesses exemplos encontram amparo em ações de políticas públicas no Brasil, como a Lei 12.608/2012 que instaura a Política Nacional de Proteção e Defesa Civil (PNPDEC) (BRASIL, 2012) e também em ações internacionais, a partir da Agenda para o Desenvolvimento Sustentável, lançada pela Organização das Nações Unidas para a Educação, a Ciência e a Cultura (UNESCO) na qual destaca a necessidade de redução do número de mortes e de populações afetadas por desastres, focando na proteção 
dos grupos mais desfavorecidos e em situação de vulnerabilidade, além de promover uma maior resiliência e uma capacidade de adaptação aos eventos que levam a uma condição de risco.

Diante do que foi apresentado esse trabalho tem como objetivo analisar a vulnerabilidade das edificações e sua distribuição espacial em parte do perímetro urbano do município de Itaoca, estado de São Paulo, que foi severamente afetado por movimentos de massa e inundações.

\section{2. Área de estudo}

Em 2014 foi registrada a ocorrência de um evento de inundações e movimentos de massa que atingiram o município de Itaoca (SP) (Figura 1), localizado no vale do rio Ribeira de Iguape. O município se estende por uma área de $183 \mathrm{Km}^{2}$ com uma população de 3.228 pessoas (IBGE, 2010) e integra a região do Alto Ribeira, marcada por um relevo predominantemente composto por topos convexos e vales estreitos, profundos e com vertentes com inclinação de 30\% a 60\% (ROSS, 2002), sendo os principais cursos hídricos o rio Palmital, que corta a área central da cidade e recebe contribuição do Guarda Mão, além do Rio Gurutuba.

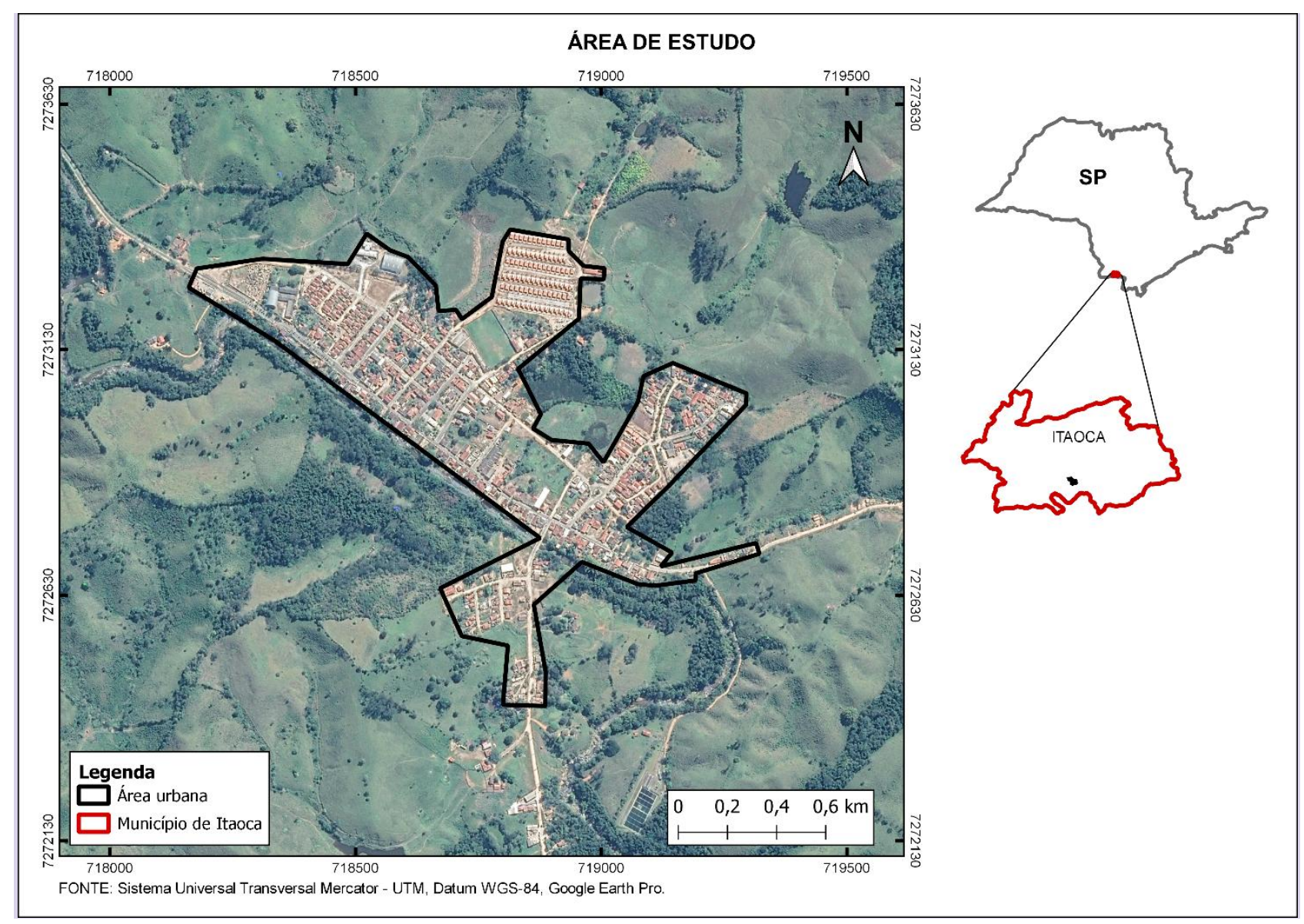

Figura 1: Mapa Localização da área de estudo. Fonte: Autor.

Segundo Brollo et al., (2015), entre os dias 12 e 13 de janeiro de 2014, ocorreu uma precipitação excessiva causando um acúmulo de aproximadamente $150 \mathrm{~mm} / 6 \mathrm{~h}$ concentrada nas cabeceiras de três bacias de drenagem - Rio Palmital, Guarda-Mão e Gurutuba - provocando a elevação repentina do nível do rio Palmital entre 4 e 5m. A intensidade da precipitação, em conjunto com o período de sua ocorrência, deflagrou escorregamentos nas vertentes, formando uma enxurrada e ocasionando o arrasto de diversos materiais e sedimentos, que provocou fluxo de detritos, de lama, bem como de vegetação e entulhos carreados ao longo do rio Palmital (BROLLO et al., 2015; CAROU et al., 2017, BONINI et al., 2017). O evento causou a morte de 25 pessoas, o desaparecimento de três, e um total de 412 construções foram atingidas pelo fluxo de lama em associação com detritos vegetais, além de sedimentos finos (areia e cascalhos) (GRAMANI, 2015; MATOS et al., 2018).

No centro da cidade de Itaoca as edificações mais afetadas foram àquelas localizadas junto às margens do rio Palmital e a montante da ponte Centro-Vila Ribas, que ao reter o material lenhoso vindo com fluxo, 
potencializou a inundação que se espalhou para as residências do bairro Vila Ribas ao entorno e a jusante da ponte (BROLLO et al., 2015; MATOS et al., 2018) (Figura 2).
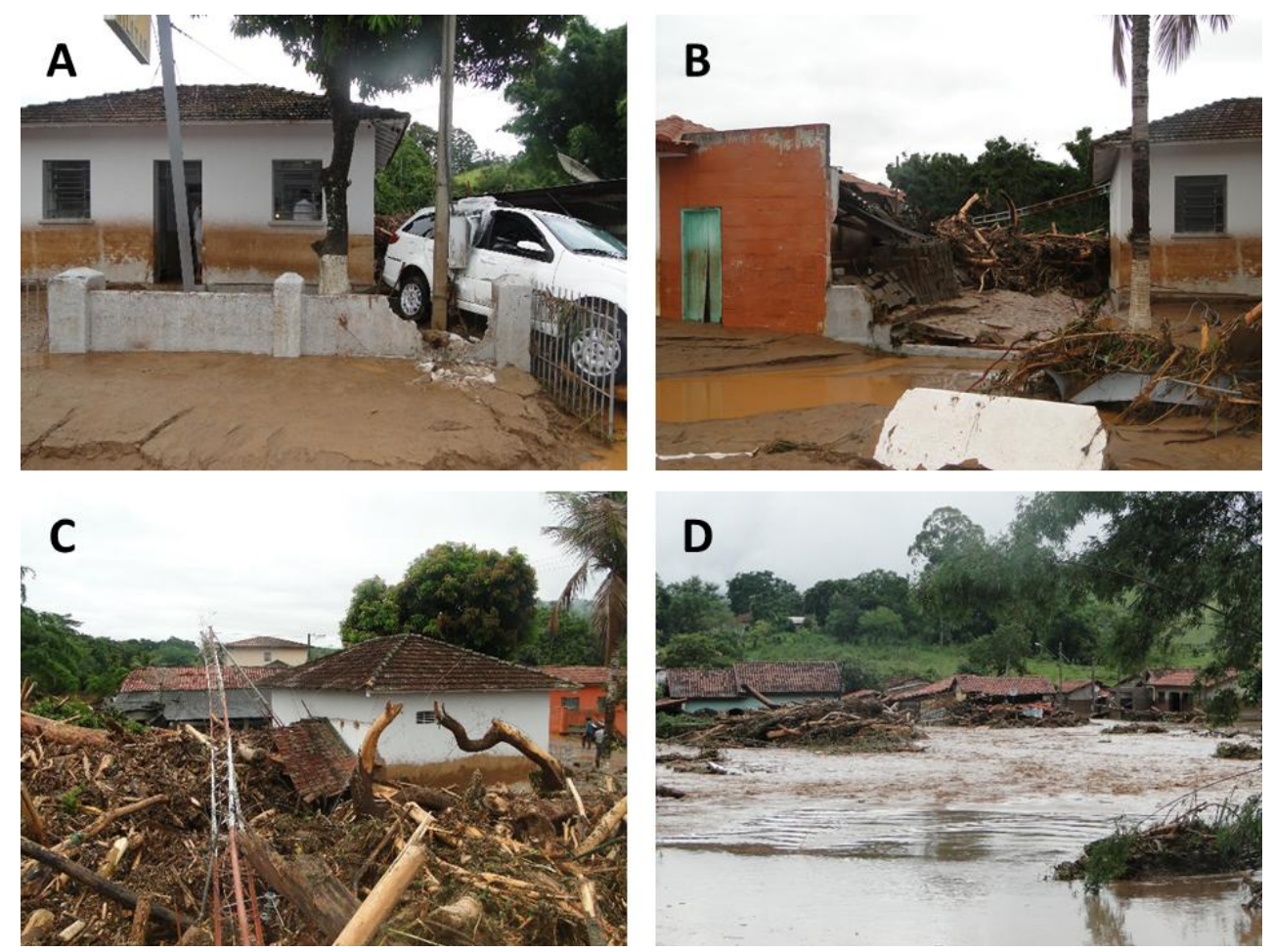

Figura 2: Área urbana do município de Itaoca, SP, afetada por fluxo de lama em 2014. A) Danos causados pela inundação. Nota-se o alcance da água dado pela marca na parede; B) Edificação destruída por detritos lenhosos; C) Acúmulo de material mobilizado pela inundação; D) Inundação que atingiu as edificações em Itaoca. Fonte: Prefeitura de Itaoca, SP.

Eventos dessa natureza foram registrados no município em anos anteriores. Em 1997 o Rio Palmital transbordou por conta de uma cheia no Rio Ribeira de Iguape, no qual desagua a jusante da cidade, porém sem atingir pontos centrais de Itaoca. Março de 1998 houve registro de quedas de barreiras e desabrigados por conta da intensidade das chuvas (BROLLO et al., 2015).

\section{Materiais e métodos}

De modo a analisar a vulnerabilidade das edificações do centro urbano de Itaoca, foi determinado um perímetro de estudo que incorporasse a Setorização de Risco feita após o evento de 2014, que classificou quatro níveis de Risco (R1: Baixo; R2: Médio; R3: Alto; R4: Muito Alto) a partir da correlação das características dos processos de movimentos de massa e seu alcance, de dados do meio físico, índices do relevo e levantamentos de campo para a elaboração de dados cartográficos em escala 1:3.000 (IG, 2015). Neste perímetro foram inventariadas 36 edificações, o que corresponde a estimados $10 \%$ do total de edificações residenciais, buscando como critério de amostragem a distribuição das edificações na área e incluindo edificações tanto dentro dos setores de risco (em todos os níveis) quanto fora, além da disposição do arruamento (Figura 3).

O inventário foi realizado em campo por meio de uma ficha que elencou as seguintes características construtivas: Número de Pavimentos, Distância do Canal; Ocorrência, ou não, de Muro de Alvenaria ao redor da edificação; Tipo de Material construtivo (Alvenaria, Composição Mista ou Madeira), tendo esses critérios sido baseados em Matos et al. (2018). O levantamento de campo foi realizado no final de setembro de 2019 e os dados foram organizados em um Sistema de Informações Geográficas (SIG).

O método escolhido para a análise da vulnerabilidade foi o PTVA (Papathoma Tsunami Vulnerability Assessment), empregado para determinar a vulnerabilidade física dos elementos de risco (pessoas, objetos, estruturas) frente a um tipo de desastre (PAPATHOMA et al., 2003). Neste trabalho, empregou-se a versão PTVA-1, na qual os elementos de risco (edificações) apresentam maior relevância para o processo deflagrado. 


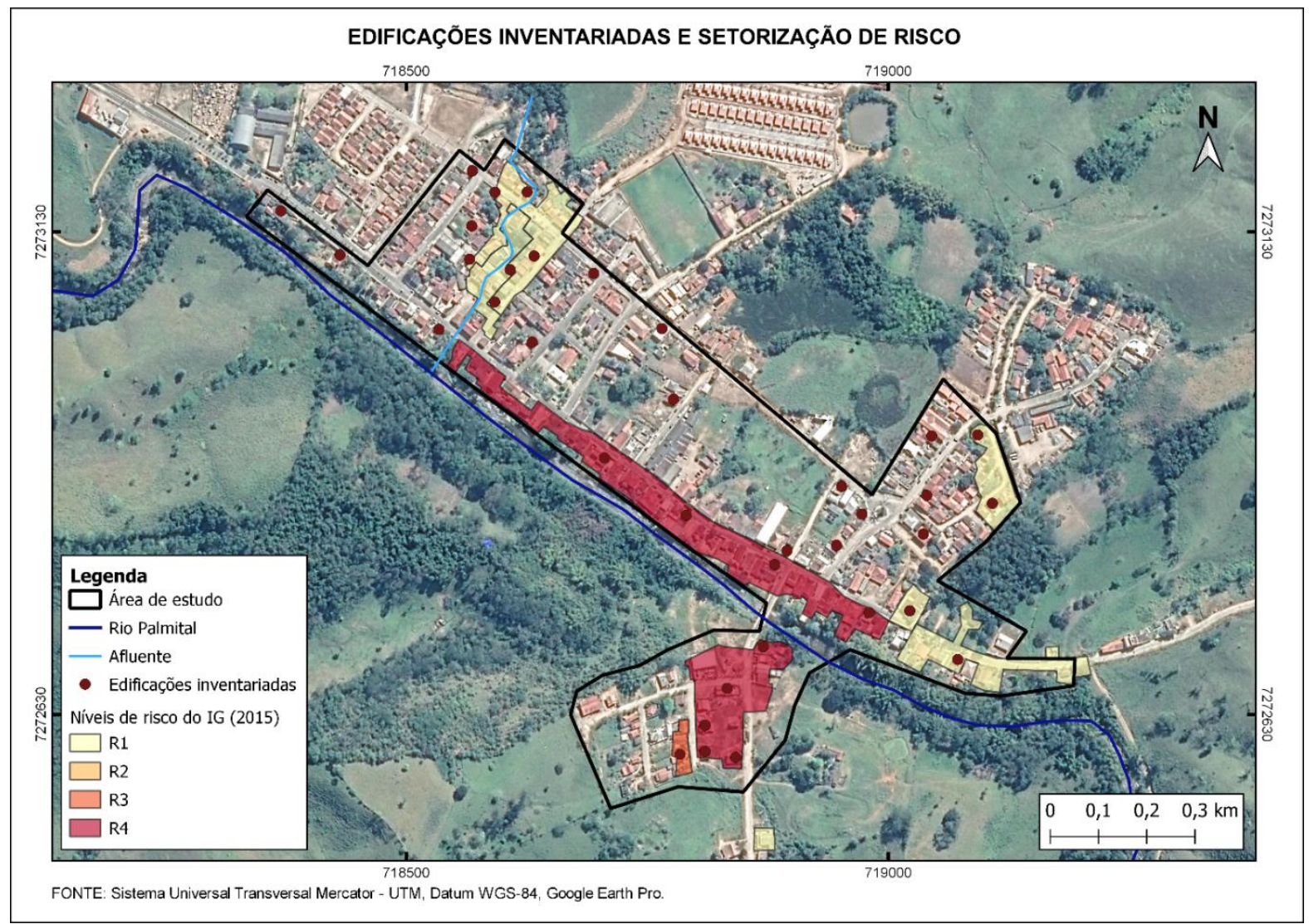

Figura 3: Edificações inventariadas e Setorização de Risco (IG, 2015).

É fundamental destacar que a proposição inicial do método PVTA foi feita para avaliar a vulnerabilidade de edificações na ocorrência de um tsunami, entretanto, o método foi atualizado para avaliar a vulnerabilidade relacionada a ocorrência de movimentos de massa por Papathoma-Köhle et al. (2007) e aplicado no Brasil, pela primeira vez, por Matos et al. (2017) no qual foi avaliada a vulnerabilidade de edificações em uma área afetada por fluxos de detritos, no município de Caraguatatuba (SP).

Dessa forma, para aplicação do método foram definidas três etapas: 1) definição dos critérios que afetam a vulnerabilidade; 2) cálculo da Avaliação de Multicritérios de Voogd; 3) representação espacial das vulnerabilidades.

Na primeira etapa, os critérios adotados foram número de pavimentos (1;2 ou mais), distância do canal hídrico ( $<30 \mathrm{~m} ;>30 \mathrm{~m}$ ), existência de muro de alvenaria (sim; não) e material da edificação (alvenaria; composição mista; madeira), em ordem crescente do critério que mais afeta a vulnerabilidade. Em cada um dos critérios cada categoria recebeu um código (1, 2 e 3), a fim de se aplicar no cálculo multicritérios.

No método de Avaliação de Multicritérios de Voogd (1983) utilizou-se o cálculo da Transformação Linear Simples, na qual, cada código foi dividido pelo número máximo de códigos para cada critério, obtendo-se o valor de padronização (Tabela 1).

Tabela 1: Critérios adotados com padronização e pesos aplicados no cálculo multicritérios

\begin{tabular}{|c|c|c|c|c|}
\hline Critérios & Descrição & $\begin{array}{l}\text { Peso } \\
\text { critério }\end{array}$ & $\begin{array}{c}\text { Contagem } \\
\text { bruta }\end{array}$ & $\begin{array}{c}\text { Contagem } \\
\text { Padronizada }\end{array}$ \\
\hline Número pavimentos & $\begin{array}{c}1 \\
2 \mathrm{ou}+\end{array}$ & 4 & $\begin{array}{l}2 \\
1\end{array}$ & $\begin{array}{c}1 \\
0.5\end{array}$ \\
\hline $\begin{array}{c}\text { Distância do canal } \\
\text { hídrico }\end{array}$ & $\begin{array}{l}<30 \mathrm{~m} \\
>30 \mathrm{~m}\end{array}$ & 3 & $\begin{array}{l}2 \\
1\end{array}$ & $\begin{array}{c}1 \\
0,5\end{array}$ \\
\hline $\begin{array}{l}\text { Muro de } \\
\text { alvenaria }\end{array}$ & $\begin{array}{l}\text { Sim } \\
\text { Não }\end{array}$ & 2 & $\begin{array}{l}1 \\
2\end{array}$ & $\begin{array}{c}0,5 \\
1\end{array}$ \\
\hline $\begin{array}{l}\text { Material } \\
\text { edificação }\end{array}$ & $\begin{array}{c}\text { Alvenaria } \\
\text { Composição mista } \\
\text { Madeira }\end{array}$ & 1 & $\begin{array}{l}1 \\
2 \\
3\end{array}$ & $\begin{array}{c}0,33 \\
0,66 \\
1\end{array}$ \\
\hline
\end{tabular}


Assim, foi atribuído um peso a cada um dos critérios, sendo 4 o peso de máximo valor, e 1 o de menor. Embora o tipo de material construtivo da edificação (Alvenaria, Composição Mista ou Madeira) apresente uma maior relevância na quantificação da vulnerabilidade associada aos movimentos de massa (MATOS et al., 2017), esse critério recebeu peso 1 (menor valor) no trabalho em tela, pois ficou evidenciado no levantamento de campo que quase a totalidade das edificações na área analisada são edificadas em alvenaria, exceto por uma, com composição mista.

Para o cálculo da Vulnerabilidade Final (VF), multiplica-se cada contagem padronizada por seu respectivo peso, obtêm-se o somatório, dividindo o resultado por 10 - a soma dos pesos dos 4 critérios (Equação 1).

$$
\mathrm{VF}=\frac{\sum((4 . a)+(3 . b)+(2 . c)+(1 . d))}{10}
$$

Onde $a, b, c$, e $d$ são a contagem padronizada de cada critério:

$a=$ número de pavimentos

$b=$ distância do canal hídrico

$c=$ muro de alvenaria

$d=$ material da edificação

Para espacializar o resultado de VF das 36 edificações amostradas para toda a área, propõe-se aqui a adoção do método de estimativa de densidade por meio de uma função kernel, que consiste em determinar a intensidade de uma ocorrência a partir da medida de distância associada a um atributo de valor conhecido (LONGLEY et al., 2013). Um exemplo de aplicação desse método para análise de vulnerabilidade pode ser encontrado em Portnov et al. (2009), que avaliaram a relação entre poluição do ar e a incidência de câncer de pulmão na área metropolitana de Haifa (Israel).

Por fim, cabe destacar que no trabalho em tela adotou-se um raio de busca de 100 metros de cada ponto inventariado para o emprego da função kernel, tendo o intervalo sido baseado nas Quebras Naturais (Jenks), em quatro classes: Baixa, Média, Alta e Muita Alta Vulnerabilidade.

\section{Resultados e discussão}

Os resultados mostram que dentre as características avaliadas, 23 das 36 edificações não possuem Muro de Alvenaria, sendo esse o critério de maior implicação na VF, uma vez que registrou o valor mais alto $(1,0)$ para um maior número de edificações. Seguido a esse, o $\mathrm{N}^{\circ}$ de Pavimentos marcou 1,0 para 8 edificações e apenas duas registraram um valor de 0,5. Já a Distância do Canal teve registro tanto em valores de 0,5 (nove) quanto de 1,0 (cinco). O critério de menor peso foi o Material Construtivo, uma vez que apenas uma edificação registrou valor acima de 0,6, enquanto as outras marcaram valores inferiores a 0,4 (Figura 4), o que é explicado pelo fato das edificações, em sua maioria, serem construídas em alvenaria.

O critério de Ocorrência de Muro circundando as edificações também teve peso significativo nos resultados apresentados por Matos et al. (2017), que avaliaram a vulnerabilidade de edificações em uma área afetada, sobremaneira, por fluxos de detritos, no município litorâneo de Caraguatatuba (SP). Os autores também destacam que o material empregado na edificação (alvenaria) reduziu o valor final da Vulnerabilidade, similarmente ao que é apresentado no trabalho em tela.

A VF para as 36 edificações revela que houve uma variação entre um mínimo de 0,48 a um valor máximo de 0,93 (Figura 4), registrando uma Média de 0,80, de Moda 0,93 e Mediana de 0,78. O resultado da Moda é bastante significativo, uma vez que 12 edificações alcançaram este alto valor $(0,93)$ enquanto apenas uma registrou um valor de 0,48. Em resumo, há uma maior quantidade de edificações com valores de VF de 0,93, implicando em uma vulnerabilidade muito elevada.

A distribuição percentual das classes do Mapa de Densidade indica que um pouco mais de $14 \%$ da área tem Baixa Vulnerabilidade, aumentando para 39\% e 35\% entre as duas classes intermediárias e, com redução para 10\% para a classe de Muito Alta Vulnerabilidade (Figura 5B).

A maior concentração de edificações com VF entre 0,73 a $>0,83$ foi no setor Norte da área, fato associado a não existência de Muros de Alvenaria e a Proximidade junto ao canal afluente do Rio Palmital, o que implicou na definição de uma Muito Alta Vulnerabilidade. Ao longo do Rio Palmital ocorrem as outras sete edificações com um maior VF (>0,83), intercaladas por duas com VF de até 0,73 (Figura 5A). De fato, a ausência de barreira de proteção nas edificações foi determinante na elevação da vulnerabilidade, havendo uma clara concentração no setor mencionado, mas com ocorrência distribuída para toda a área avaliada (Figura 6). 


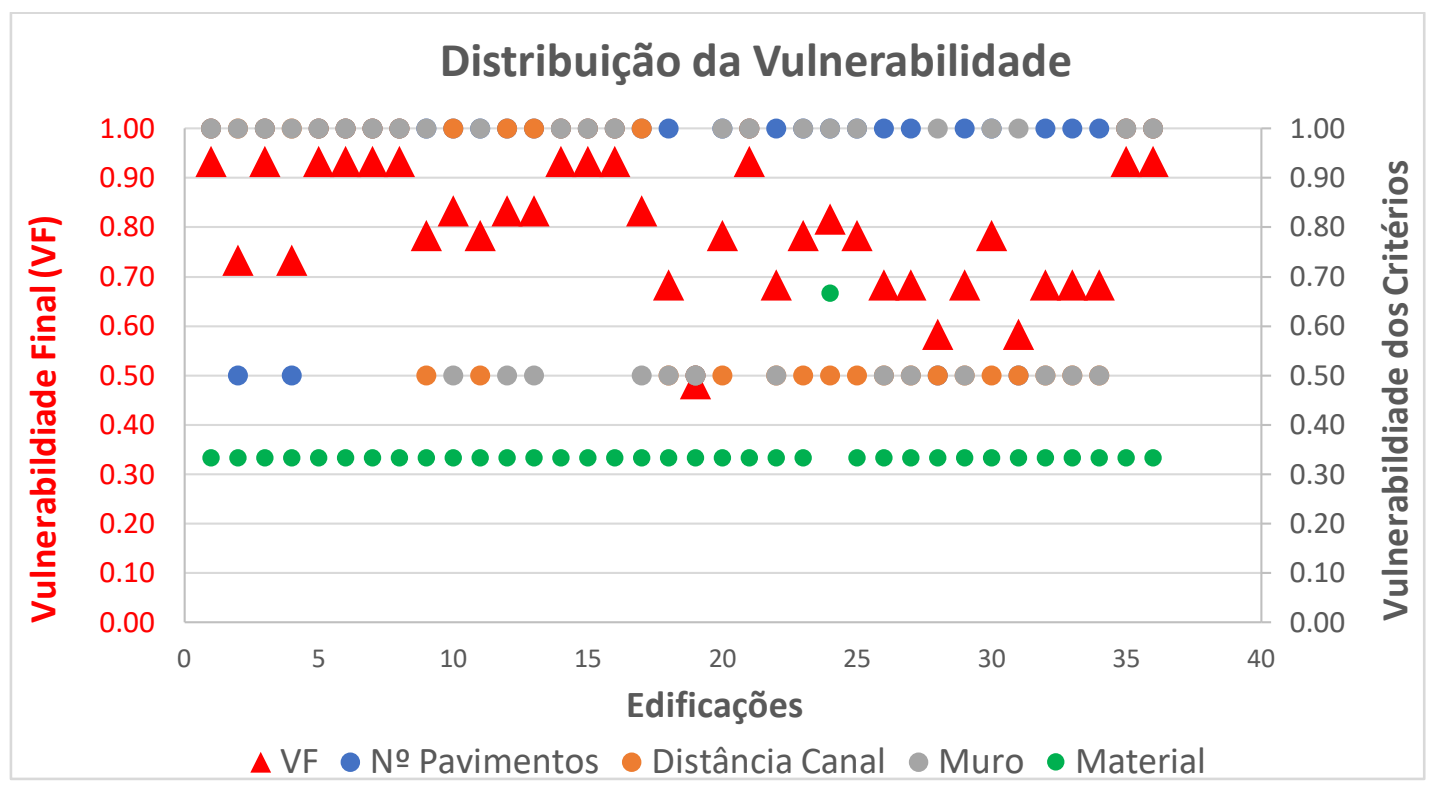

Figura 4: Distribuição da Vulnerabilidade Final e da Vulnerabilidade de cada critério avaliado.

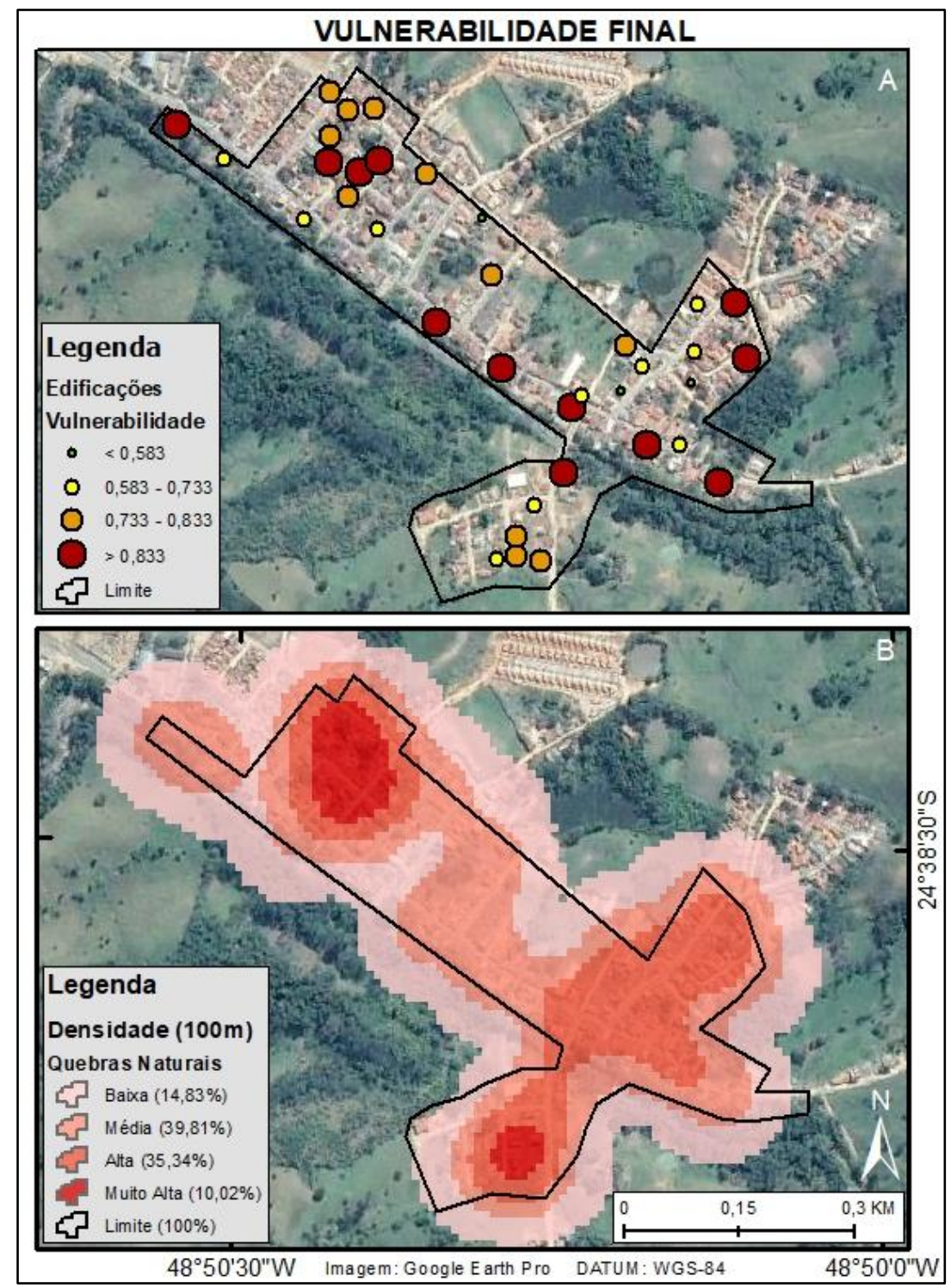

Figura 5: A) Edificações inventariadas com o resultado da Vulnerabilidade e B) Mapa de Densidade da vulnerabilidade das edificações. 
O Mapa de Densidade (Figura 5B) se mostra distinto da Setorização de Risco elaborado por IG (2015) (Figura 3). Naquele trabalho, por exemplo, é destacado um setor de Risco Muito Alto (R4) ao longo da margem esquerda do Rio Palmital, enquanto é definido um Setor de Risco Baixo (R1) para a área ao Norte, o que é o oposto do mapa aqui apresentado. Todavia, há uma coincidência entre um setor R4 e Muito Alta Vulnerabilidade no setor Sul da área (Figura 5B).

Deve-se destacar que a maior parte das edificações inventariadas ao longo do Rio Palmital também registraram, individualmente, altos valores de Vulnerabilidade, contudo, diante do espaçamento entre elas, a densidade foi menor. Contrariamente, o espaçamento das edificações no setor Norte é menor e, ainda, ocorrem outras edificações com vulnerabilidade $>0,73$, ou seja, há um maior número de edificações com vulnerabilidade variando entre >0,73 a >0,83 (Figura 5A), em uma área menor.
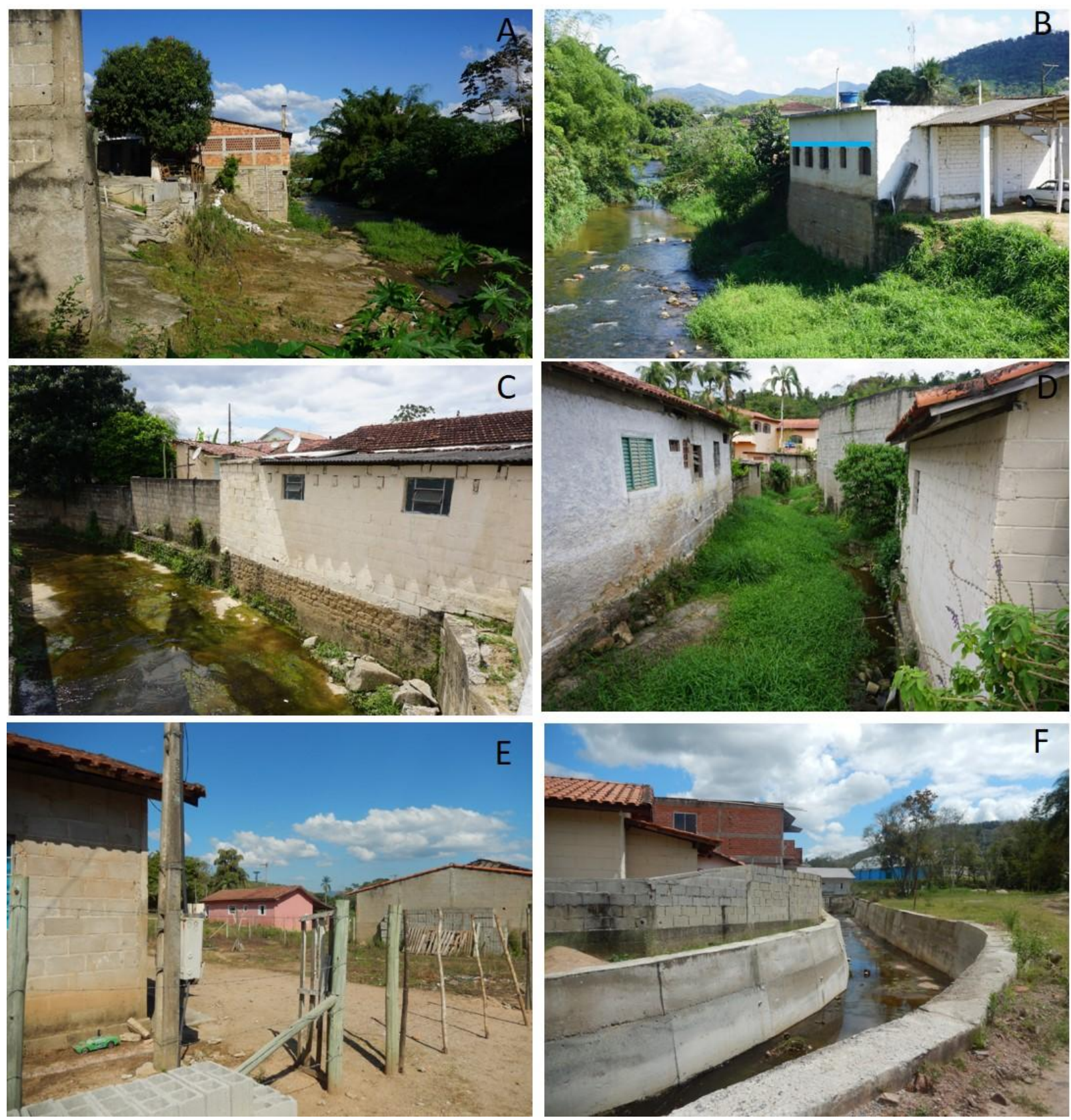

Figura 6: Exemplos dos tipos de edificações presentes na área de estudo. A e B) Edificações às margens do Rio Palmital (nota-se a ausência de muro), a linha em azul indica o alcance da inundação de 2014; C e D) Edificações às margens do afluente do Palmital, uma área com a maior concentração de Muito Alta Vulnerabilidade; E) Edificação no Setor Sul, nota-se a ausência de muros; F) Edificação às margens do afluente, nota-se a presença de muro de alvenaria. 
O fato de haver uma Baixa Vulnerabilidade para as edificações situadas ao longo do Rio Palmital, conforme indicado pelo Mapa de Densidade (Figura 5B) não é indicativo de ausência de vulnerabilidade, como revelam os valores individuais de cada edificação, lembrando que não houve nenhuma edificação com valor de Vulnerabilidade $<0,48$. Deste modo, mesmo as faixas de Baixa Vulnerabilidade no Mapa da Figura 5B indicam uma vulnerabilidade significativa. Esse entendimento se associa aos resultados de Matos et al. (2018) que inventariam as edificações danificadas pelo evento de $2014 \mathrm{em}$ Itaoca, indicando que várias delas foram danificadas justamente ao longo do Rio Palmital. E encontra par no trabalho de Almeida et al. (2016) que, ao analisarem um índice de exposição a desastres naturais, qualificaram como baixa exposição os valores superiores a 0,55 , numa classificação que varia até 1 .

Em suma, o Mapa de Densidade da vulnerabilidade das edificações evidencia uma Muito Alta Vulnerabilidade nas edificações mais adensadas, construídas junto ao afluente do rio Palmital e sem muro circundante, o que se repete ao Sul da área e em menor intensidade nas demais, sendo o critério de ocorrência de muro o mais significativo no resultado.

Um ponto de destaque é que a maior densidade de vulnerabilidade (Figura 5B) possui uma forte associação com a ocorrência de lotes de menor tamanho circundando a edificação, ao passo que mesmo àquelas edificações com maior vulnerabilidade, a exemplo daquelas localizadas ao longo do Rio Palmital, com valores individuais $>0,83$ (Figura 5A), demonstraram uma espacialização de menor valor da vulnerabilidade, o que pode ser explicado diante da distância entre as amostras, o que é um reflexo da dimensão do tamanho dos lotes. Por fim, mesmo em não havendo coincidência direta com os níveis de risco da setorização (IG, 2015), a menor Vulnerabilidade não anula a condição do nível de Risco mapeado e, tampouco um menor nível de risco, implica em uma menor Vulnerabilidade.

\section{Considerações finais}

O emprego dos métodos PTVA e a análise da espacialização pelo Mapa de Densidade mostraram-se importantes ferramentas para gerar cartografia de risco, de forma a serem utilizados no planejamento urbano e em casos de necessidade emergencial devido a praticidade e brevidade na aplicação, mediante anterior decisão dos critérios a serem adotados e seus respectivos pesos no cálculo da Vulnerabilidade Final (VF). Ademais, torna-se relevante evidenciar que a avaliação de campo é crucial na elaboração da Ficha de Inventário, bem como na decisão dos fatores de maior influência na vulnerabilidade.

Desse modo, o resultado denota que apesar dos desastres ocorridos anteriormente a área ainda apresenta significativa vulnerabilidade a novos eventos. Assim, salienta-se a preocupante condição dos habitantes de Itaoca que estão sujeitos a danos sociais e materiais recorrentes, sendo perceptível a necessidade prioritária de se adotar medidas mitigatórias e planos de ação Municipal e Estadual em conjunto com a Defesa Civil, a fim de se preparar para lidar com o desastre e diminuir os impactos ocasionados por processos naturais, porém, intensificados pelo desenvolvimento humano. Essas ações paliativas serão responsáveis por atenuar prejuízos socioeconômicos, de forma a se possibilitar investimentos em outras áreas, e ainda, proteger os habitantes.

Futuramente podem ser realizados na cidade de Itaoca estudos que determinem a implantação de intervenções construtivas para reduzir a vulnerabilidade das edificações mais propensas a novos fluxos de detrito e lama, tal como pesquisas que visem a gestão de riscos a movimentos de massa e planos que norteiem o desenvolvimento urbano local.

\section{Agradecimentos}

Os autores agradecem ao CNPq pela concessão de bolsa de Iniciação Científica (PIBIC-UNIFESP). Agradecem a Geógrafa Dr. Luzia Matos (PPGF-USP) pelas recomendações de leituras, a Profa. Dra. Bianca C. Vieira (USP) e ao Geógrafo Msc. Evandro Daniel (PPGF-USP) pelo apoio no trabalho de campo. Ao Sr. Junior, Secretário de Turismo do Município de Itaoca pela recepção e ao Dr. Cláudio Ferreira do Instituto Geológico (SP), pela cessão dos dados vetoriais dos Setores de Risco. Estendem seus agradecimentos aos moradores de Itaoca por acolherem os pesquisadores em visita e aproveitam para cumprimentar e agradecer aos Editores e Revisores da Revista do Departamento de Geografia pelas críticas e sugestões. 
Referências

ALMEIDA, L.T.; WELLE, T. BIRKMANN, J. Disaster Risk Indicators in Brazil: A Proposal Based On The World Risk Index. International Journal of Disaster Risk Reduction, v. 17. p. 251-272. 2016.

BRASIL. Ministério das Cidades / Instituto de Pesquisas Tecnológicas - IPT. Mapeamento de Riscos em Encostas e Margem de Rios. CARVALHO, C.S. MACEDO, E.M. OGURA, A.T. (org.) - Brasília: Ministério das Cidades; Instituto de Pesquisas Tecnológicas - IPT, 2007176 p.

BRASIL, LEI No 12.608, DE 10 DE ABRIL DE 2012. 2012.

BROLlO, M. J.; CARLOS, A.; GUEDES, M. Desastres Naturais E Riscos Em São Luiz Do Paraitinga (Sp). 7o Simpósio Brasileiro de Cartografia Geotécnica e Geoambiental. Anais...2010

BROLlO, M. J.; SANTORO, J.; PENTEADO, D. R.; FERNANDES DA SILVA, P. C.; RIBEIRO, R. R. Itaóca (SP): Histórico de acidentes e desastres relacionados a perigos geológicos. 14 $^{\circ}$ Simpósio de Geologia do Sudeste. Campos do Jordão - SP: 1-5. 2015.

BONINI, J. E.; VIEIRA, B. C.; MARTINS, T.D.; GRAMANI, M. F. Parâmetros Morfológicos e os Escorregamentos na Bacia do Rio Gurutuba, Vale do Ribeira (SP). In: PEREZ FILHO, A.; AMORIM, R.R. (Org.). Os Desafios da Geografia Física na Fronteira do Conhecimento. 1ed.Campinas: Unicamp, v. 1, p. 4011-4019. 2017.

BONINI, J.E.; ROSS, J.L.S.; VIEIRA, B.C.; MARTINS, T.D. Escorregamentos rasos em São Luiz do Paraitinga (SP, Brasil) (2009-2010). Physis Terrae - Revista Ibero-Afro-Americana de Geografia Física e Ambiente, v. 2, p. 85-99, 2021.

CAROU, C.; VIEIRA, B.C.; MARTINS, T.D.; GRAMANI, M. Inventário dos Escorregamentos da Bacia do Rio Gurutuba, Vale do Ribeira (SP). Revista Do Departamento De Geografia, p.172-179. 2017.

CERRI, L.E.S.; NOGUEIRA, F.R.; CARVALHO, C.S.; MACEDO, E.S.; AUGUSTO FILHO, O. Mapeamento de risco em assentamentos precários no município de São Paulo (SP). Geociências, v. 26, n. 2, p.143-150. 2007.

CRED. Centre for Research on the Epidemiology of Disasters. Economic Losses, Poverty \& Disasters 1998-2017. UNISDR. 33p. Disponível em: https://www.cred.be/publications. 2018.

DE PLOEY, J. e CRUZ, O. Landslides in the serra do mar, Brazil. Catena, 6(2), pp.111-122. 1979. DOI: 10.1016/0341-8162(79)90001-8.

DIAS, V.C.; VIEIRA, B. C.; GRAMANI, M. F. Parâmetros morfológicos e morfométricos como indicadores da magnitude das corridas de detritos na Serra do Mar Paulista. Confins (Paris), v. 29, p. 1-20, 2016.

DIAS, M.C.A.; SAITO, S.M.; ALVALÁ, R.C.S.; SELUCHI, M.E.; BERNARDES, T.; CAMARINHA, P.I.M.; STENNER, C.; NOBRE, C.A. Vulnerability index related to populations at-risk for landslides in the Brazilian Early Warning System (BEWS). International Journal of Disaster Risk Reduction, Volume 49. 2020 .

FERNANDES, N.F.; GUIMARÃES, R.F.; GOMES, F.A.T.; VIEIRA, B.C.; MONTGOMETY, D.R.; GREENBERG, H. Condicionantes Geomorfológicos dos Deslizamentos nas Encostas: avaliação de metodologias e aplicação de modelo de previsão de áreas susceptíveis. Revista Brasileira de Geomorfologia, v.2, n.1, p.51-71, 2001.

GRAMANI, M. F. A corrida de massa no córrego Guarda-Mão, Município de Itaoca (SP): Impacto e observações de campo. In: Congresso Brasileiro de Geologia de Engenharia e Ambiental, Bento Gonçalves. Anais do $15^{\circ}$ Congresso Brasileiro de Geologia de Engenharia. São Paulo: ABGE, Cd-Rom, 2015.

GRAMANI, M. F.; ARDUIN, D. H. Morfologia da drenagem dos depósitos de debris flow em Itaóca, São Paulo. $15^{\circ}$ Congresso Brasileiro de Geologia de Engenharia e Ambiental. In: Congresso Brasileiro de 
Geologia de Engenharia e Ambiental, Bento Gonçalves. Anais do $15^{\circ}$ Congresso Brasileiro de Geologia de Engenharia. São Paulo: ABGE, Cd-Rom, p.10. 2015.

GUILLARD-GONÇALVES, C.; ZÊZERE, J.L. Combining Social Vulnerability and Physical Vulnerability to Analyse Landslide Risk at the Municipal Scale. Geosciences 8, 2018.

IBGE. Instituto Brasileiro de Geografia e Estatística. Censo 2010. Disponível em: https://cidades.ibge.gov.br/brasil/sp/itaoca/panorama/.

IBGE. Instituto Brasileiro de Geografia e Estatística. População em Áreas de Risco no Brasil. Rio de Janeiro. 2018. Disponível em: https://www.ibge.gov.br/apps/populacaoareasderisco/.

IG. INSTITUTO GEOLÓGICO (IG-SMA). Mapeamento de riscos associados a escorregamentos, inundações e corridas de massa - Município de Itaoca, SP. São Paulo: Instituto Geológico, Secretaria do Meio Ambiente do Estado de São Paulo. Relatório Técnico, 2015. 3 volumes.

LONGLEY, P.A.; GOODCHILD, M.F.; MAGUIRE, D.J.; RHIND, D.W. Sistemas e Ciência da Informação Geográfica. 3ed. Porto Alegre: Bookman. 2013. 540p.

MACEDO, E. S. de; MARTINS, P. P. D. Análise do banco de dados de mortes por deslizamentos do Instituto de Pesquisas Tecnológicas (IPT). In: CONGRESSO BRASILERIO DE GELOGIA DE ENGENHARIA E AMBIENTAL, 15.,2015, Bento Gonçalves. Anais... São Paulo: ABGE, 2015. cd-rom. 7p.

MATOS, L. J.; VIEIRA, B. C.; FERREIRA, C. J. Avaliação da vulnerabilidade de construções a corridas de detritos por meio do método PTVA (Papathoma Tsunami Vulnerability Assessment). Boletim Paulista de Geografia, v. 1, p. 42-65, 2017.

MATOS, L.; FERREIRA, C.; BATEIRA, C.; VIEIRA, B. Avaliação das Construções Danificadas por Corridas de Detritos e Inundações Bruscas no Vale do Ribeira (SP) em 2014. Revista do Departamento de Geografia, p. 57-67. 2018.

PAPATHOMA, M.; DOMINEY.H, D.; ZONG, Y.; SMITH.D. Assessing Tsunami vulnerability, an example from Herakleio, Greece. Natural Hazards and Earth System. Sciences, 3, 377-389. 2003.

PAPATHOMA-KÖHLE, M., NEUHÄUSER, B., RATZINGER, K., WENZEL, H., and DOMINEYHOWES, D.: Elements at risk as a framework for assessing the vulnerability of communities to landslides, Natural Hazards and Earth System Sciences, Vol.7, p. 765-779. 2007.

PICANÇO, J. L., \& NUNES, L. H. 2013. A severe convective episode triggered by accumulated precipitation in the coast of Parana State, Brazil. In Proceedings of 7th European conference of severe storms. pp. 4-14. 2013.

PORTNOV, B.A.; DUBNOV, J.; BARCHANA, M. Studying the association between air pollution and lung cancer incidence in a large metropolitan area using a kernel density function. Socio-Economic Planning Sciences. v. 43 (3). p141-150. 2009.

ROSS, J. A morfogênese da bacia do Ribeira do Ribeira do Iguape e os sistemas ambientais. GEOUSP Espaço e Tempo, São Paulo, N 12, p. 21 - 46, 2002.

VIEIRA, B.C.; GRAMANI, M. Serra Do Mar: The Most “Tormented" Relief In Brazil. In: (Eds) VIEIRA, B.C, SALGADO, A.A.R.; SANTOS, L.J.C. Landforms and Landscapes of Brazil. Springer. Pp. 285-298. 2015. DOI: 10.1007/978-94-017-8023-0_26.

VOOGOD, H. Multicriteria evaluation with mixed qualitative and quantitative data. International Institute for applied systems analysis. Laxenburg, Austria, 1983.

ZAI, C.; GRASSO, L.; SILVEIRA, C.T.; GUARNIERI, D.P. Impactos Socioambientais por Movimentos de Massa na Comunidade de Floresta, Região da Serra Do Mar Paranaense. Caminhos de Geografia, v. 18, n. 61, p. 19-32, 2017. 
$102 \begin{aligned} & \text { Este artigo é distribuído nos termos e condições do Creative Commons Attributions/Atribuição- } \\ & \text { NãoComercial-CompartilhaIgual (CC BY-NC-SA). }\end{aligned}$ 